\title{
Distribución y susceptibilidad a fluconazol de levaduras del género Candida aisladas en pacientes hospitalizados y ambulatorios
}

\author{
Claudio Alburquerque O., Germán Hermosilla D. y Cecilia Tapia P.
}

\section{Species distribution and fluconazole susceptibility of yeasts of genus Candida isolated from hospitalized and ambulatory patients}

Most surveillance studies have included invasive candidiasis from hospitalized patients. However, no national study has evaluated the species distribution and susceptibility to fluconazole of Candida species isolated from hospitalized and ambulatory patients. A total of 166 strains were collected consecutively during a 6 month period. Strains were isolated from vaginal fluid $(73.5 \%)$, urine $(7.8 \%)$, lower respiratory tract samples $(7.8 \%)$, blood cultures $(4.2 \%)$, sterile fluids $(2.4 \%)$ and wounds $(1.8 \%)$. Most of the isolates were obtained from ambulatory patients $(71.1 \%)$. The species found were Candida albicans $(78.9 \%)$, C. glabrata $(8.4 \%)$, C. tropicalis $(6.0 \%)$, C. famata (1.8\%), C. krusei (1.8\%), C. parapsilosis (1.8\%) and C. sake (1.2\%). Fluconazole susceptibility was: 92.3\% for C. albicans, $85.7 \%$ for $C$. glabrata (most strains being dose-dependent susceptible), $100 \%$ for $C$. parapsilosis and $80 \%$ for $C$. tropicalis. Only susceptible strains were isolated from hospitalized children, whereas more resistant strains were isolated from ambulatory adults, mainly from vaginal fluid. In order to identify probable reservoirs of less susceptible strains such as C. glabrata, it would be necessary to include ambulatory isolates in future surveillance studies.

Key words: Species distribution, Candida, fluconazole, ambulatory, reservoir.

Palabras clave: Distribución de especies, Candida, fluconazol, ambulatorio, reservorio.

\section{Introducción}

$\mathrm{D}$ esde 1980 los hongos han emergido como importantes oportunistas, que afectan especialmente a pacientes inmunocomprometidos ${ }^{1,2}$. Dentro de los agentes productores de micosis en este tipo de pacientes, la especie más frecuentemente aislada es Candida albicans; sin embargo, otras especies como $C$. glabrata, C. parapsilosis, C. tropicalis y C. krusei han emergido como agentes causales de candidiasis, siendo algunas de ellas resistentes a algunos antifúngicos ${ }^{2,3}$. En estudios de vigilancia chilenos se ha descrito un aumento del aislamiento de especies no-albicans en los últimos años en infecciones fúngicas invasoras ${ }^{4,5}$. Estos datos concuerdan con otros estudios de vigilancia en Norteamérica y Latinoamérica, donde se ha observado que las especies de Candida no-albicans, presentan cambios epidemiológicos continuos en su frecuencia con una tendencia al aislamiento de especies más resistentes a antifúngicos ${ }^{2,6}$.

A pesar de que estos cambios se han descrito en candidiasis invasora y afectan a pacientes internados en unidades críticas, también se han detectado cambios en pacientes de otros servicios clínicos y en pacientes de la comunidad $^{2,7,8}$.

Es importante destacar que los reportes epidemiológicos se han realizado en candidiasis invasora ${ }^{6-11} \mathrm{y}$ en algunas candidiasis superficiales como candidiasis orofaríngea en pacientes con $\operatorname{SIDA}^{12,13}$ y candidiasis vulvovaginal, por separado ${ }^{14,15}$.

Clínica Dávila es una institución que atiende tanto a pacientes hospitalizados como ambulatorios. Recibe en promedio 2.300 cultivos mensuales, de los cuales $68,8 \%$ corresponden a urocultivos, $23, \%$ a cultivos corrientes, $6,2 \%$ a flujos vaginales y $1,8 \%$ a cultivos de hongos, aislándose del total de cultivos mensuales entre 25 y 30 levaduras del género Candida ( $1 \%$ de aislamiento).

No existen estudios que describan la distribución de especies y susceptibilidad a fluconazol de cepas de Candida aisladas tanto en pacientes hospitalizados como ambulatorios, por lo que en este estudio se realiza un análisis global de todas las cepas obtenidas en un período de seis meses considerando el tipo de muestra y los datos demográficos de los pacientes.
Clínica Dávila, Santiago, Chile Unidad de Microbiología (CAO, CTP) Facultad de Medicina, Universidad de Chile, Santiago Instituto de Ciencias Biomédicas Programa de Microbiología y Micología (CAO, GHD, CTP)

Fuente de Financiamiento: BecaPG/50/2005, Depto. de Postgrado y Postítulo, Universidad de Chile

Recibido: 23 de junio de 2008 Aceptado: 20 de abril de 2009

Correspondencia a: Cecilia Tapia Paredes cecilia.tapia@davila.cl 


\section{Material y Métodos}

Cepas: Se estudiaron 166 aislados pertenecientes a un cepario recolectado entre enero y junio de 2007, de manera consecutiva, abarcando todas las cepas de Candida aisladas de muestras clínicas de pacientes hospitalizados y ambulatorios atendidos en Clínica Dávila. Se consideraron pacientes hospitalizados aquellos que se encontraran internados en alguno de los servicios de Clínica Dávila al momento de la toma de muestra del cultivo y como ambulatorios, aquellos que consultaron en el Centro Médico de la institución. Se obtuvieron retrospectivamente los antecedentes demográficos generales de los pacientes, los cuales fueron analizados manteniendo en estricta confidencialidad su identidad. Se realizó un análisis descriptivo de los aislados de acuerdo al tipo de muestra, al sexo y a la edad de los pacientes.

Las cepas fueron descongeladas y sembradas en agar Sabouraud glucosado e incubadas 24 a $48 \mathrm{hrs}$ a $37^{\circ} \mathrm{C}$.

Identificación de levaduras del género Candida: Las especies de levaduras se identificaron según procedimientos empleados en micología médica, analizando las características fisiológicas de las levaduras (tubo germinal y microcultivo), agar cromogénico (ChromAgar Candida $\left.{ }^{\circledR}\right)^{16}$ y API ID $32 \mathrm{C}{ }^{17}$.

Medición de la concentración inhibitoria mínima: Las cepas congeladas en glicerol fueron sembradas en agar Sabouraud glucosado e incubadas por $18 \mathrm{hrs}$. Se midieron las CIMs de las colonias aisladas a fluconazol mediante microdilución en caldo del estándar europeo del European Committee on Antimicrobial Susceptibility Testing-Subcommittee on Antifungal Susceptibility Testing (EUCAST-ASFT) ${ }^{18}$ utilizando como control las cepas C. krusei ATCC 6258 y C. parapsilosis ATCC 22019.

Cada cepa fue clasificada frente a cada fármaco como sensible (S), sensible dosis dependiente (SDD) y resistente $(\mathrm{R})$, según los criterios establecidos por el estándar del Clinical and Laboratory Standards Institute (CLSI) documento M27-A2 ${ }^{20}$ (Tabla 1).

Análisis estadístico: Se realizó con la prueba chi cuadrado $\left(\chi^{2}\right)$ con una significancia estadística de $\mathrm{p}<0,05$ utilizando el programa STATA 7.0

\section{Resultados}

El $71,1 \%$ de las cepas de Candida se aislaron de pacientes ambulatorios y $28,9 \%$ de pacientes hospitalizados. Del total de pacientes $84,3 \%$ correspondieron a mujeres y $15,7 \%$ a hombres. La mayoría de las levaduras se aislaron de adultos $(\mathrm{n}=157)$ y sólo 9 aislados pertenecieron a niños, siendo la mayoría de los niños de edad inferior a un año.

Se recuperó Candida de flujo vaginal $(73,5 \%)$, orina $(7,8 \%)$, muestras respiratorias bajas $(7,8 \%)$, hemocultivos $(4,2 \%)$, líquidos estériles $(2,4 \%)$ y heridas $(1,8 \%)$. Las muestras no agrupadas en las anteriores correspondieron a $2,4 \%$.

El mayor porcentaje de levaduras recuperadas en mujeres fue obtenido de flujo vaginal y sólo $12,9 \%$ correspondió a otros sitios. De un total de 44 muestras no vaginales, $26(59,0 \%)$ correspondieron a aislados provenientes de hombres y 18 (41,0\%) de mujeres. En hombres se aisló Candida con mayor frecuencia de muestras respiratorias bajas $(34,6 \%)$ que en mujeres $(22,2 \%)$, mientras que en mujeres, se aisló Candida más frecuentemente en orina $(38,8 \%)$ respecto a hombres $(23,1 \%)(p<0,05)$ (Tabla 2$)$.

Las localizaciones más frecuentes en pacientes bajo un año de edad fueron sangre $(57,2 \%)$ y orina $(42,8 \%)$.

\begin{tabular}{|c|c|}
\hline \multicolumn{2}{|c|}{$\begin{array}{c}\text { Tabla 1. Puntos de corte para fluconazol de } \\
\text { Candida sp, de acuerdo al documento M27-A2 } \\
\text { del estándar del CLSI* }\end{array}$} \\
\hline Categoría & Fluconazol $(\mu \mathrm{g} / \mathrm{ml})$ \\
\hline Sensible & $\leq 8$ \\
\hline Sensible dosis dependiente & $16-32$ \\
\hline Resistente & $\geq 64$ \\
\hline
\end{tabular}

\begin{tabular}{|lcc|}
\hline \multicolumn{2}{|c|}{ Tabla 2. Distribución de muestras con aislados } \\
de Candida sp según sexo \\
Muestras & Mujeres & Hombres \\
Flujo vaginal & 122 & - \\
Otras muestras: & 18 & 26 \\
& $\mathbf{n}(\%)^{*}$ & $\mathbf{n ~ ( \% ) * ~}$ \\
Respiratoria baja & $4(22,2)$ & $9(34,6)$ \\
Orina & $7(38,8)$ & $6(3,6)$ \\
Sangre & $2(11,1)$ & $5(3,0)$ \\
Líquido estéril & $1(5,6)$ & $3(1,8)$ \\
Herida & $1(5,6)$ & $2(1,2)$ \\
Otros & $3(16,7)$ & $1(0,6)$ \\
\hline * Porcentaje calculado respecto a muestras no vaginales en mujeres \\
y en hombres por separado. & & \\
\hline
\end{tabular}


Sin considerar los flujos vaginales, en los adultos las muestras más frecuentes fueron respiratorias bajas y orinas (Tabla 3), abarcando 59,0\% de las muestras (26/44).

Del total de cepas analizadas $78,9 \%$ correspondieron a Candida albicans, 8,4\% a C. glabrata, 6,0\% a C. tropicalis, $1,8 \%$ a C. famata, $1,8 \%$ a $C$. krusei, $1,8 \%$ a $C$. parapsilosis y $1,2 \%$ a C. sake. Candida glabrata fue la segunda especie en frecuencia y se aisló preferentemente de flujo vaginal y orina, mientras que $C$. parapsilosis fue aislada con mayor frecuencia de sangre.

Tanto en pacientes ambulatorios como hospitalizados, los rangos de susceptibilidad global de las cepas a fluconazol estuvieron entre $0,125 \mu \mathrm{g} / \mathrm{mL}$ y $64 \mu \mathrm{g} / \mathrm{mL}$. Los porcentajes de susceptibilidad a fluconazol fueron: $92,3 \%$ en C. albicans, $85,7 \%$ en C. glabrata (de las cuales, $78,6 \%$ fueron sensibles dosis dependiente), $100 \%$ en $C$. parapsilosis y $80 \%$ en C. tropicalis (Tabla 4). En todos los

\begin{tabular}{|c|c|c|c|c|}
\hline \multirow{3}{*}{ Muestra } & \multicolumn{4}{|c|}{ Edad (años) } \\
\hline & $0-1$ & $1-15$ & $16-50$ & $>50$ \\
\hline & n $(\%)^{*}$ & n $(\%)^{*}$ & n (\%)* & n $(\%)^{*}$ \\
\hline Respiratoria baja & $\begin{array}{ll}0 & (0,0)\end{array}$ & $0 \quad(0,0)$ & $3(33,4)$ & $10 \quad(38,5)$ \\
\hline Orina & $3(42,8)$ & $(0,0)$ & $3(33,4)$ & $\begin{array}{ll}7 & (26,9)\end{array}$ \\
\hline Sangre & $4 \quad(57,2)$ & $(0,0)$ & $1(11,1)$ & $2 \quad(7,6)$ \\
\hline Líquidos estériles & $(0,0)$ & $(0,0)$ & $0 \quad(0,0)$ & $4 \quad(15,4)$ \\
\hline Heridas & $(0,0)$ & $0 \quad(0,0)$ & $1(11,1)$ & $1 \quad(3,8)$ \\
\hline Otros & $0 \quad(0,0)$ & $1(100,0)$ & $1(11,1)$ & $2 \quad(7,7)$ \\
\hline Total & $7(100,0)$ & $1(100,0)$ & $9(100,0)$ & $26(100,0)$ \\
\hline
\end{tabular}

\section{Tabla 4. Distribución de susceptibilidad a fluconazol de las especies de Candida según el tipo de muestra}

\begin{tabular}{|c|c|c|c|c|c|c|c|c|c|}
\hline Especie & Categoría & Vaginal & Herida & $\begin{array}{l}\text { Líquido } \\
\text { estéril }\end{array}$ & Orina & Respiratoria baja & Sangre & Otras & $\begin{array}{c}\text { Total } \\
\text { n } \quad(\%)\end{array}$ \\
\hline \multirow[t]{3}{*}{ C. albicans } & S & 98 & 1 & - & 8 & 8 & 4 & 2 & $121 \quad(92,3)$ \\
\hline & SDD & 7 & 1 & - & 1 & - & - & - & $9 \quad(6,8)$ \\
\hline & R & 1 & - & - & - & - & - & - & $1 \quad(0,8)$ \\
\hline \multirow[t]{3}{*}{ C. glabrata } & s & 1 & - & - & - & - & - & - & $1 \quad(7,1)$ \\
\hline & SDD & 8 & - & - & 2 & 1 & - & - & $11(78,6)$ \\
\hline & $\mathrm{R}$ & 2 & - & - & - & - & - & - & $2(14,3)$ \\
\hline \multirow[t]{3}{*}{ C. tropicalis } & S & 2 & 1 & 2 & 1 & 2 & - & - & $8 \quad(80,0)$ \\
\hline & SDD & - & - & - & - & - & - & - & $\begin{array}{ll}0 & (0,0)\end{array}$ \\
\hline & R & - & - & 1 & - & 1 & - & - & $2(20,0)$ \\
\hline \multirow[t]{3}{*}{ C. famata } & S & - & - & - & - & - & - & - & $\begin{array}{ll}0 & (0,0)\end{array}$ \\
\hline & SDD & - & - & - & - & - & - & - & $(0,0)$ \\
\hline & R & 1 & - & 1 & 1 & - & - & - & $3(100,0)$ \\
\hline \multirow[t]{3}{*}{ C. krusei } & S & - & - & - & - & - & - & - & $(0,0)$ \\
\hline & SDD & - & - & - & - & - & - & - & $0 \quad(0,0)$ \\
\hline & $\mathrm{R}$ & 1 & - & - & - & 1 & 1 & - & $3(100,0)$ \\
\hline \multirow[t]{3}{*}{ C. parapsilosis } & S & - & - & - & - & - & 2 & 1 & $3(100,0)$ \\
\hline & SDD & - & - & - & - & - & - & - & $(0,0)$ \\
\hline & R & - & - & - & - & - & - & - & $(0,0)$ \\
\hline \multirow[t]{3}{*}{ c. sake } & S & 1 & - & - & - & - & - & 1 & $2(100,0)$ \\
\hline & SDD & - & - & - & - & - & - & - & $0 \quad(0,0)$ \\
\hline & $\mathrm{R}$ & - & - & - & - & - & - & - & $(0,0)$ \\
\hline
\end{tabular}


pacientes bajo 15 años de edad se aislaron cepas sensibles, mientras que todas las cepas resistentes se obtuvieron en pacientes adultos, de los cuales, 5/9 cepas resistentes $(55,5 \%)$ procedían de flujo vaginal.

\section{Discusión}

Dado que las infecciones fúngicas han aumentado el último tiempo concomitantemente con un aumento de pacientes de riesgo, su vigilancia, provee importante información sobre la incidencia y las tendencias de estas infecciones. Estos estudios de vigilancia se realizan usualmente en infecciones fúngicas invasoras, sin considerar la presencia de levaduras del género Candida en infecciones superficiales, que pueden representar potenciales reservorios de infecciones invasoras.

En nuestro estudio, la mayoría de los aislados de levaduras del género Candida se recuperaron de pacientes ambulatorios $(71,1 \%)$, fundamentalmente de muestras vaginales. Aunque los flujos vaginales sólo corresponden a $6,2 \%$ del total de cultivos, el aislamiento de Candida es mayor en este tipo de muestra, por tratarse de un microorganismo comensal.

Un estudio reciente demostró que las cepas de Candida aisladas de pacientes con infección del tracto urinario en unidades de cuidados intensivos eran clonalmente idénticas a las obtenidas de muestra vaginal ${ }^{21}$, lo cual, apoya la idea de que la mayoría de las infecciones por Candida en pacientes hospitalizados son endógenas, lo cual podría explicar la mayor prevalencia de candiduria en mujeres, respecto a hombres en este estudio (Tabla 2).

Otros autores han hecho ver la importancia de vigilar también las infecciones fúngicas en pacientes ambulatorios que, aunque no comprometen la vida del paciente, representan la microbiota presente, pudiendo traducirse en una infección invasora si se presentan las condiciones de una infección oportunista ${ }^{2}$.

Sin considerar las muestras de flujo vaginal, los dos tipos de muestras que continúan en frecuencia son las respiratorias bajas y las de orina (Tabla 2), aisladas fundamentalmente de pacientes hospitalizados. Aunque el rol patógeno de levaduras en muestras respiratorias bajas es discutido, pues las neumonías por Candida son inusuales ${ }^{22}$, en pacientes hospitalizados la vía respiratoria podría actuar como reservorio del agente.

La segunda especie aislada, en frecuencia, después de C. albicans, fue C. glabrata $(8,4 \%)$, recuperada principalmente de flujos vaginales. Otras especies aisladas con menor frecuencia fueron C. tropicalis $(6,0 \%), C$. krusei $(1,8 \%)$, C. famata $(1,8 \%)$ y C. parapsilosis $(1,8 \%)$; esta última se recuperó exclusivamente de muestras pediátricas (niños bajo un año de edad), lo que se ha descrito previamente en otros estudios ${ }^{5}$.
En relación a la susceptibilidad a fluconazol, la mayoría de las cepas de C. albicans fueron sensibles a fluconazol, mientras que $C$. glabrata mostró una menor susceptibilidad a fluconazol, siendo $76,8 \%$ de las cepas sensibles dosis dependiente (SDD).También la mayoría de estas cepas se aislaron de flujo vaginal. Candida parapsilosis no mostró resistencia a fluconazol lo que coincide con un estudio nacional previo ${ }^{5}$. Candida tropicalis mostró un porcentaje no despreciable de resistencia (20\%) lo cual había sido descrito previamente ${ }^{5}$. Tanto, C. krusei como C. famata aisladas en este estudio presentaron $100 \%$ de resistencia a fluconazol. La resistencia de C. krusei es intrínseca a azoles, mientras que ocasionalmente cepas de C. famata pueden presentar resistencia primaria a estos antifúngicos ${ }^{2,21}$.

En todos los pacientes bajo 15 años de edad, los aislados fueron sensibles a fluconazol. En pacientes adultos se aislaron muestras resistentes, fundamentalmente de flujo vaginal. La microbiota vaginal es un reservorio importante de levaduras del género Candida; por lo tanto, debiera considerarse la vigilancia de este tipo de infecciones.

Este estudio muestra la realidad de una institución que atiende tanto a pacientes hospitalizados como ambulatorios, comprobándose que la mayor parte de las muestras desde donde se aíslan levaduras del género Candida provienen de pacientes ambulatorios, adultos y corresponden principalmente a flujos vaginales presentando algunas especies menor susceptibilidad a fluconazol, como $C$. glabrata, C. krusei y C. famata (Tabla 4).

La distribución de especies y la resistencia a azoles, varía de acuerdo al sexo, edad y procedencia de los pacientes. El conocimiento de la distribución de especies y susceptibilidad a azoles en pacientes ambulatorios podría ser útil, considerando que podrían representar reservorios de infecciones fúngicas invasoras. Estudios futuros, que incluyan el análisis de la historia clínica de los pacientes ambulatorios y un seguimiento de estos, podrán intentar comprobar esta hipótesis.

\section{Resumen}

La mayoría de los estudios de vigilancia de levaduras del género Candida se realizan en candidiasis invasora en pacientes hospitalizados; sin embargo, no existen estudios que evalúen simultáneamente la distribución global de especies y susceptibilidad a fluconazol de cepas de Candida aisladas de pacientes hospitalizados y ambulatorios. Se analizaron 166 cepas de Candida, recolectadas consecutivamente en un período de 6 meses, las cuales fueron aisladas de flujo vaginal $(73,5 \%)$, orina $(7,8 \%)$, muestras respiratorias bajas $(7,8 \%)$, hemocultivos $(4,2 \%)$, líquidos estériles $(2,4 \%)$ y heridas $(1,8 \%)$. Un $71,1 \%$ provenía de pacientes ambulatorios. La especies aisladas 
fueron Candida albicans (78,9\%), C. glabrata (8,4\%), C. tropicalis $(6,0 \%)$, C. famata $(1,8 \%), C$. krusei $(1,8 \%), C$. parapsilosis $(1,8 \%)$ y C. sake $(1,2 \%)$. Los porcentajes de sensibilidad a fluconazol fueron: $92,3 \%$ para $C$. albicans, $85,7 \%$ para C. glabrata (siendo la mayoría sensible dosis dependiente), $100 \%$ para $C$. parapsilosis y $80 \%$ para $C$. tropicalis. En los niños, todos hospitalizados, se aislaron sólo cepas sensibles, mientras que en adultos ambulatorios se aislaron más cepas resistentes, fundamentalmente de flujo vaginal. Considerar cepas ambulatorias en futuros estudios de vigilancia permitirían dar cuenta de probables reservorios de cepas más resistentes, como C. glabrata.

\section{Referencias}

1.- Calderone R A. Candida and Candidiasis. Editorial ASM Press, Washington DC. 2002. Págs. 37-53.

2.- Pfaller M A, Diekema D J. Epidemiology of invasive candidiasis: a persistent public health problem. Clin Microbiol Rev 2007; 20: 133-63.

3.- Martínez-Suárez J V, Rodríguez-Tudela J L. La resistencia a antifúngicos en los hongos patógenos oportunistas (II). Imidazoles y triazoles. Enferm Infecc Microbiol Clin 1996; 14: 490-8.

4.- Silva V, Díaz M C, Febré N, and Chilean invasive infection group. Invasive fungal infections in Chile: a multicenter study of fungal prevalence and susceptibility during a 1-year period. Med Mycol 2004; 42: 333-9.

5.- Alvarado D, Díaz M C, Silva V. Identificación y susceptibilidad antifúngica de Candida spp. aisladas de micosis invasora. Influencia del porcentaje de inhibición del crecimiento para la determinación de la CIM. Rev Méd Chile 2002; 130; 4: 416-23.

6.- Pfaller M A, Jones R N, Doern G, Sader H S, Hollis R J, Messer S A, for the Sentry Participant Group. International surveillance of bloodstream infections due to Candida species: frequency of occurrence and antifungal susceptibilities of isolates collected in 1997 in the United States, Canada, and South America for the SENTRY Program. J Clin Microbiol 1998; 36: 1886-9.

7.- Hajjeh R A, Sofair A N, Harrison L H, Lyon G M, Arthington-Skaggs B A, Mirza S A, et al. Incidence of bloodstream infections due to Candida species an in vitro susceptibilities of isolates collected from 1998 to 2000 in a population-based active surveillance program. J Clin Microbiol 2004; 42: 1519-27.
8.- Kao A, Brandt M, Pruitt W R, Conn L, Perkins B, Stephens D, et al. The epidemiology of candidemia in two United States cities: result of population-based active surveillance. Clin Infect Dis 1999; 29: 1164-70.

9.- Rodero L, Davel G, Córdoba S, Soria M, Cantero C, Hochenfeller F. Multicenter study on nosocomial candidiasis in the Republic of Argentina. Rev Argent Microbiol 1999; 31: 114-19.

10.- Pfaller M A, Jones R N, Doern G V, Sader H S, Messer S A, Houston A, et al, and The Sentry Participant Group. Bloodstream infections due to Candida species: SENTRY Antimicrobial Surveillance Program in North America and Latin America, 1997-1998. Antimicrob Agents Chemother 2000; 44: 747-51.

11.- Colombo A L. Epidemiology and treatment of hematogenous candidiasis: a Brazilian perspective. Braz J Infect Dis 2000; 3: 113-8.

12.- Barchiesi F, Arzeni D, Del Prete M S, Sinico A, Falconi di Francesco L, Pasticci M B, et al. Fluconazole susceptibility and strain variation of Candida albicans isolates from HIV-infected patients with oropharyngeal candidosis. J Antimicrob Chemother 1998; 41: 541-8.

13.- Walmsley S, King S, McGeer A, Ye Y, Richardson S. Oropharyngeal candidiasis in patients with immunodeficiency virus: correlation of clinical outcome with in vitro resistance, serum azole levels and immunosuppression. Clin Infect Dis 2001; 32: 1554-61.

14.- Sobel J D, Zervos M, Reed B D, Hooton T, Soper D, Nyirjesky P, et al. Fluconazole susceptibility of vaginal isolates obtained from women with complicated Candida vaginitis: clinical implications. Antimicrob Agents Chemother
2003; 47: 34-8.

15.- Sobel J D. Vulvovaginal candidosis. Lancet 2007; 369: 1961-71.

16.- Odds F C, Bernaerts R. CHROMagar Candida, a new differential isolation medium for presumptive identification of clinically important Candida species. J Clin Microbiol 1994; 32: 1923-9.

17.- Ramani R, Gromadzki S, Pincus D H, Salkin I F, Chaturvedi. Efficacy of API 20C and ID 32C systems for identification of common and rare clinical yeasts isolates. J Clin Microbiol 1998; 36: 3396-8.

18.- Subcommittee on Antifungal Susceptibility Testing (ASFT) of the ESCMID European Committee for Antimicrobial Susceptibility Testing (EUCAST). Method for the determination of minimum inhibitory concentration (MIC) by broth dilution of fermentative yeasts. Clin Microbiol Infect 2003; 9: 1-8.

19.- EUCAST Technical Note on fluconazole. The European Committee on Antimicrobial Susceptibility Testing-Subcommittee on Antifungal Susceptibility Testing (EUCAST-AFST). Clin Microbiol Infect 2008; 14: 193-5.

20.- National Committee for Clinical Laboratory Standards. (2002). Reference method for broth dilution antifungal susceptibility testing of yeasts. Approved standard NCCLS document M27-A2. National Committee for Clinical Laboratory Standards, Wayne, Pa.

21.- Silva V, Hermosilla G, Abarca C. Nosocomial candiduria in women undergoing urinary catheterization. Clonal relationship between strains isolated from vaginal tract and urine. Med Mycol 2007; 45: 645-51.

22.- Hidalgo A. Radiología de las infecciones fúngicas invasoras del aparato respiratorio. Rev Iberoam Micol 2007; 24: 14-18. 